Ann. Biol. anim. Bioch. Biophys., I970, 10 (3), 5Ir-5I5.

NOTE TECHNIQUE

\title{
INHIBITION DE LA SPERMATOGENÈSE DU GUPPY (POISSON CYPRINODONTIDAE) PAR LE MÉTHALLIBURE
}

\author{
R. BILLARD, B. BRETON et B. JALABERT \\ avec la collaboration technique de Anne-Marie EscAFFrE \\ Station centrale de Physiologie animale, \\ Centre national de Recherches zootechniques, 78 -Jouy-en-Josas \\ Institut national de la Recherche agronomique
}

\section{RÉSUMÉ}

L'utilisation du méthallibure, en bain, aux doses de I à 2 p.p.m., entraîne une inhibition de la spermatogenèse du Guppy, qui est plus lente que celle obtenue après hypophysectomie chirurgicale ; après 30 jours de traitement 1'inhibition de la spermatogenèse n'est pas totale. Le taux de mortalité reste élevé (20 p. Ioo au $30^{\circ}$ jour à la dose de I p.p.m.) mais il est dû en partie au solvant utilisé.

\section{INTRODUC'TION}

Le méthallibure ( $\alpha$-méthylallyl-thiocarbamoyl-2 méthyl-thiocarbamoyl-hydrazine $=$ I. C. I. 33 828), qui est capable d'inhiber la fonction gonadotrope hypophysaire chez certains Mammifères (PAget et al., I96I ; Schmidt-Elmendorff, I962; Bell et al., I962 ; Harper, I964; WalPole, I968; Garbers et First, r969), peut également altérer la gamétogenèse des Amphibiens (GaNgadhara et Ramaiah, I968) et des Poissons (Hoar et al, ig67 ; Wiebe, I968).

Chez les Mammifères, la similitude entre les effets de l'hypophysectomie et du méthallibure sur la spermatogenèse (HEMsworTh et al., 1968) fait supposer que l'action du méthallibure se situe au niveau des cellules gonadotropes hypophysaires. Chez les Poissons, une étude ultrastructurale de l'hypophyse aboutit au même résultat (LEATHERLAND, I969), ce qui permet d'envisager un traitement au méthallibure comme technique d'hypophysectomie. Le présent travail, réalisé dans ce but, permet une comparaison de l'influence du mode d'administration du méthallibure (par injection ou dans l'eau de l'aquarium) sur les différentes catégories de cellules germinales chez le Guppy mâle (Poecilia reticulata PETERs) et une confrontation avec des résultats déjà enregistrés après hypophysectomie chirurgicale (Billard, I969 $b$; PANDEY, I969). 


\section{MATÉRIEL ET MÉTHODES}

Le matériel animal et ses conditions d'élevage ont été décrits précédemment (BiLlarD, I969 a). Les animaux pesant entre 60 et I Io $\mathrm{mg}$ sont répartis en lots de Io dans des aquariums en chlorure de polyvinyle contenant I litre d'eau ; le contenu de ces aquariums est renouvelé tous les 2 jours par de l'eau prélevée dans un grand bassin où vivent d'autres Guppies et des plantes aquatiques. Le méthallibure est maintenu en suspension dans l'eau à l'aide du Triton $\times$ Ioo (une goutte pour Io $\mathrm{ml}$ de solution mère contenant $\mathrm{I} g$ de méthallibure).

\section{Administration par injection}

Deux lots d'animaux reçoivent respectivement tous les 3 et 6 jours une injection de I $\mu \mathrm{l}$ contenant $0, \mathrm{I} \mathrm{mg}$ de méthallibure, par animal; un lot témoin reçoit tous les 3 jours une injection de I $\mu$ l d'eau physiologique.

\section{Administration dans l'eau des aquaviums}

Les animaux sont répartis en 2 lots soumis à 2 concentrations différentes I et 2 p.p.m. La dose est renouvelée tous les 2 jours.

\section{Estimation de l'effet}

L'influence du traitement est mesurée par la mortalité (qui est contrôlée tous les jours), le rapport gonado-somatique (RGS) et l'analyse quantitative des différentes catégories cellulaires contenues dans le testicule. Dans chaque lot 2 mâles sont sacrifiés aux 1oe, $20^{\mathrm{e}}$ et $30^{\mathrm{e}}$ jours de traitement et les gonades sont fixées dans le Bouin Hollande pendant 3 jours et coupées en totalité en coupes sériées de ro $\mu$. Les comptages de cystes sont réalisés selon le processus déjà décrit (BILlARD, I969 b) et les résultats sont exprimés en nombre de cystes par apex.

\section{RÉSULTATS}

\section{Taux de mortalité}

Les résultats résumés dans le tableau I font apparaître un taux de mortalité plus élevé lorsque le méthallibure est administré par injection. La mortalité reste élevée dans les bains où

\section{TABLEAU I}

Pourcentage de mortalité aux $10^{\mathrm{e}}$, $20^{\mathrm{e}}$ et $30^{\mathrm{e}}$ jour de traitement dans les lots recevant du méthallibure

et dans le lot témoin recevant des injections d'eau physiologique

P. 100 de mortalité au:

\begin{tabular}{|c|c|c|c|}
\hline & $10^{\mathbf{e}}$ jour & $20 \mathrm{e}$ jour & $30 \mathrm{e}$ jour \\
\hline Bains : Faible dose (1 p.p.m.).. & 10 & 10 & 20 \\
\hline Forte dose (2 p.p.m.) ....... & 20 & 30 & 40 \\
\hline Témoin (Triton seul) $\ldots \ldots \ldots$ & 0 & 0 & 10 \\
\hline Injections: Faible dose $(17 \gamma / \mathrm{j})$. & 10 & 30 & 30 \\
\hline Forte dose $(33 \gamma / \mathrm{j}) \ldots \ldots \ldots \ldots$ & 30 & 40 & 60 \\
\hline Témoin (triton seul) $\ldots \ldots \ldots$ & 0 & 0 & 10 \\
\hline
\end{tabular}


la dose est la plus faible (I p.p.m.). Cependant, la comparaison avec les animaux témoins recevant seulement le solvant montre que ce dernier est partiellement responsable de la mortalité observée aussi bien dans le cas des bains que des injections.

\section{Action sur la spermatogenèse}

Dans tous les cas, il n'y a pas de différence entre les doses élevées et les doses faibles, de sorte que les résultats sont regroupés et que la comparaison porte sur le mode d'administration : bains ou injections.

L'évolution du RGS apparaît dans la figure I A. L'administration par injection entraine une diminution plus rapide, mais moins importante que dans le cas d'administration par bain.

La diminution des différentes catégories cellulaires est reproduite dans la figure I (B à $H$ ). Les premières générations spermatogoniales $\left(G_{1}-G_{7}\right)$ ne semblent pas affectées par le méthallibure administré par injection, alors que l'administration par bain diminue le nombre de cystes de cette catégorie (fig. I B). Toutes les autres cellules germinales sont affectées par le traitement, mais dans tous les cas la diminution est plus forte lorsque l'administration est faite par bain.
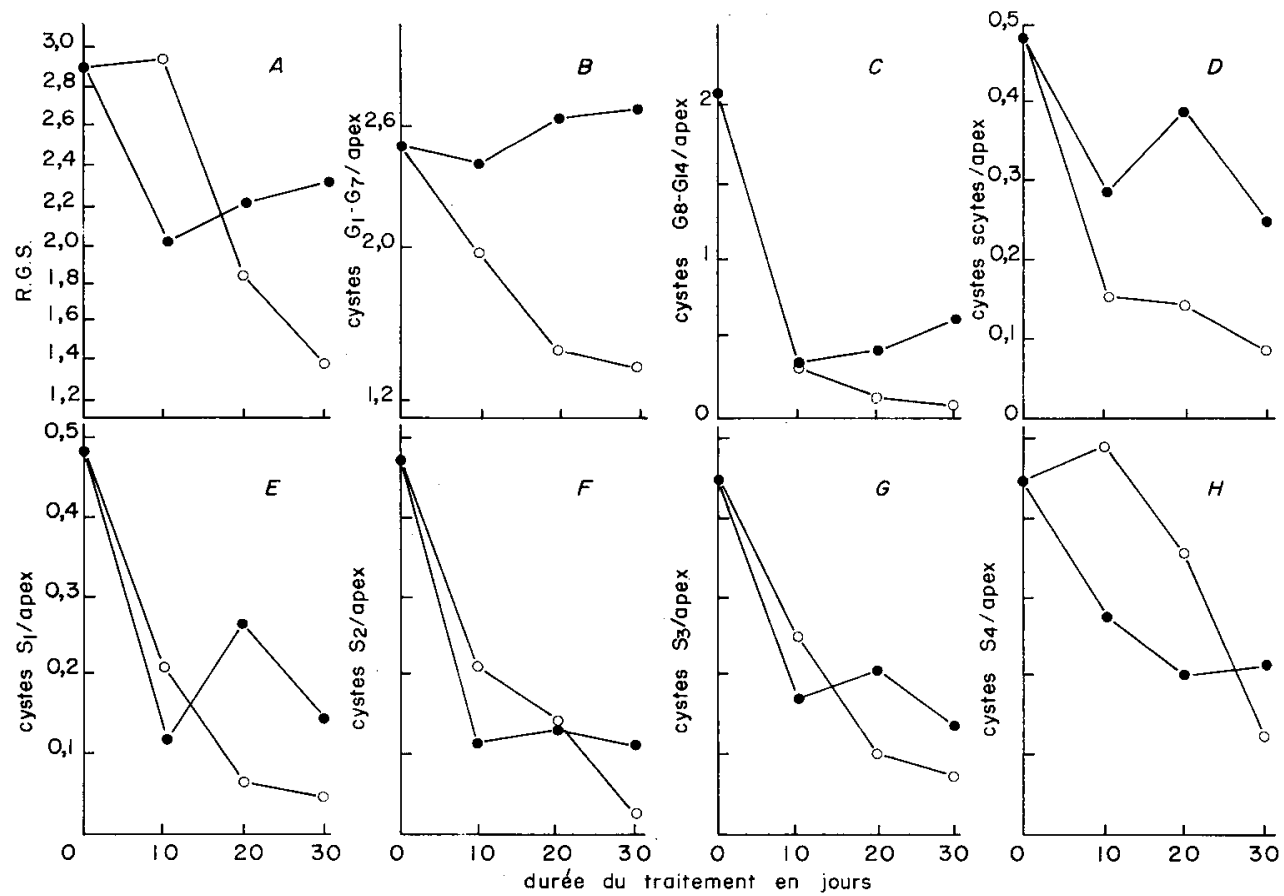

FIG. I. - Evolution de la spermatogenèse du Guppy après traitement au méthallibure

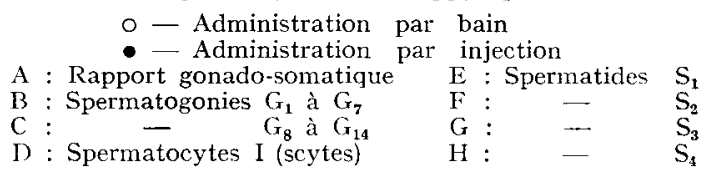

\section{DISCUSSION}

Le méthallibure entraîne une diminution importante du RGS et affecte la plupart des cellules germinales, mais l'importance des dégénérescences varie suivant le mode d'administration. L'ad- 
ministration par injection entraîne une dégénérescence rapide de la plupart des cellules germinales pendant les ro premiers jours, mais la stabilisation et, quelquefois même la restauration qui interviennent ensuite sont vraisemblablement dues à une perte du matériel injecté après une série réitérée d'injections, phénomène déjà signalé chez les Poissons (Frisen, I967).

La comparaison avec les résultats obtenus après hypophysectomie chirurgicale (BrLlard, 1969) montre que les effets du méthallibure sont beaucoup plus lents. Après 30 jours de traitement au méthallibure, le RGS est de $I, 2$, valeur qui est enregistrée Io jours seulement après hypophysectomie. Le même décalage se retrouve au cours de la régression des spermatogonies et des spermatocytes I. Cependant, l'évolution des différents types de spermatides est comparable dans les 2 cas (hypophysectomie et méthallibure), au cours des Io premiers jours. Au-delà du Ioe jour de traitement au méthallibure, Ia diminution est moins rapide et au $30^{\mathbf{e}}$ jour, il subsiste encore des cystes de chaque catégorie de spermatides, alors que 30 jours après hypophysectomie, il ne subsiste pratiquement plus de spermatides. Il semble donc qu'après 30 jours de traitement le méthallibure n'entraîne pas, aux doses utilisées, un blocage complet de l'activité gonadotrope hypophysaire.

\section{CONCLUSIONS}

Chez le Guppy, l'utilisation du méthallibure ajouté dans l'eau de l'aquarium ne constitue pas un moyen d'inhibition de l'activité gonadotrope hypophysaire aussi complet que l'hypophysiotonie chirugicale.

Reçu pour publication en avril 1970.

\section{REMERCIEMENTS}

Nous remercions la firme Impérial Chemical Industries qui nous a aimablement fourni le méthallibure utilisé dans les expériences.

\section{SUMMARY}

\section{INHIBITORY EFFECT OF ICI 33828 (METHALL,IBURE) ON SPERMATOGENESIS IN THE GUPPY (PCECILIA RETICULATA)}

Administration of methallibure at $\mathbf{I}$ to 2 p.p.m. rates in water inhibited spermatogenesis in the Guppy. The gonadosomatic index decreased after day 10 of treatment (fig. I A). A regression of the various germinal cells was also recorded, but at day $3^{\circ}$ of treatment, the inhibition of spermiogenesis was not total (fig. r B.H). In all cases the effects were slower than those recorded after hypophysectomy. Methallibure was less effective when injected than when administered in the water.

Mortality rate was high (table $\mathrm{I}$ ), but it was partly due to the solvent used ( $X$ roo Triton).

\section{RÉFÉRENCES BIBLIOGRAPHIQUES}

Bell E. T., Brown J. B., Fortheby K., Loraine J. A., Robson J. S., 1962. The effect of derivatives of dithiocarbamoyl hydrazine on hormone excretion in post menopausal women. J. Endocr., $26,425-436$. 
Billard R., Ig69a. La spermatogenèse de Poecilia reticulata. I. Estimation du nombre de générations goniales et rendement de la spermatogenèse. Ann. Biol. anim., Bioch. Biophys., 9, 25I-27I.

Billard R., I969 b. Hypophysectomie et spermatogenèse chez Poecilia reticulata (Poisson Cyprinodontidae). C. R. Acad. Sci., sér. D, 268, 1856-1859.

Frisen L., I967. Reliability of intraperitoneal injection in fish. Experientia, 23, 883.

Gangadhara W. S., Ramaiah T. R., ig68. Effect of hypophysectomy, ovariectomy, starvation and methallibure on the skipper frog, Rana cyanophlyctis. Indian J.exp. Biol., 6, 218-220.

Garbers D. L., First N. L., r 969 . The effects of injected œestradiol i $7 \beta$ progesterone and dietary I. C. I. 33828 on ovarian and pituitary functions in the sow and gilt. J. Reprod. Fert., 20, 45I-464.

HARPER M. J. K., I964. Effects of I $\alpha$ methylallylthiocarbamoyl-2-methyl thiocarbamoylhydrazine (I. C. I. 33828) on early pregnancy in the rat. J. Reprod. Fert., 7, 2 II-220.

Hoar W. S., Wiebe J., WAI E. H., r967. Inhibition of the pituitary gonadotropin activity of fishes by a dithiocarbamoyl hydrazine derivative (I. C. I. 33828), Gen. Comp. Endocr., 8, roI-rog.

Hemsworth B. N., Jackson H., Walpole A. L., I968. Effect of the pituitary inhibitor I. C. I. 33828 (methallibure) on the reproductive system of male rats. J. Endocr., 40, 275-284.

LEATHERLAND J. F., I969. Studies on the structure and ultrastructure of the intact and "methallibure "treated meso-adenohypophysis of the Viviparous Teleost Cymatogaster aggregata GiBbons. Z. Zellforsch., 98, 122-134.

Paget G. E., Walpole A. L., Richardson D. N., I96I. Non steroidal inhibitors of pituitary gonadotrophic function. Nature, 192, II9I.

PANDEY S., 1969. Effects of hypophysectomy on the testis and secondary sex characters of the adult guppy Poecilia reticulata PETERS. Can. J. Zool, 47, 775-781.

Schmidt-ElmendorfF M., Loraine J. A., Bell E. T., Walley J. K., Ig62. An investigation into the effect of derivatives of dithiocarbamoyl hydrazine on gonadotrophic activity in the body fluids on pregnant mares. J. Endocr., 25, I07-1 14 .

WAlpole A. L., I968. Non steroidal drugs in relation to ovulation and implantation. J. Reprod. Fert., suppl. 4, 3-I 4.

WIEBE J. P., rg68. Inhibition of pituitary gonadotropic activity in the viviparous searperch $C y m a t o-$ gaster aggregata GiBBoNs by a dithiocarbamoylhydrazine derivative I. C. I. 33828. Can. J. Zool., 46, $75 \mathrm{I}-758$.

\section{NOTE AJOUTÉE SUR ÉPREUVES}

Martin et Bromage (I969. J. Fish. Biol. 2. 47-5I) dans une étude analogue sur la même espèce observent une diminution significative dans le nombre de spermatocytes et de spermatides après administration de méthallibure mais ces 2 catégories cellulaires n'ont pas complètement disparu au bout de 5 semaines de traitement. 